\title{
Prediction of Unified New Physics beyond Quantum Mechanics from the Feynman Path Integral: Elementary Cycles String Theory
}

Donatello Dolce ( $\sim$ donatello.dolce@unicam.it)

University of Camerino https://orcid.org/0000-0002-5761-5933

\section{Research Article}

Keywords: Feynman Path Integral, elementary particles, particle internal clock, quantum mechanics

Posted Date: May 20th, 2021

DOl: https://doi.org/10.21203/rs.3.rs-503118/v1

License: (c) (i) This work is licensed under a Creative Commons Attribution 4.0 International License.

Read Full License 
Noname manuscript No.

(will be inserted by the editor)

\title{
Prediction of Unified New Physics beyond Quantum Mechanics from the Feynman Path Integral \\ Elementary Cycles String Theory
}

\author{
Donatello Dolce
}

the date of receipt and acceptance should be inserted later

\begin{abstract}
We prove that the Feynman Path Integral is equivalent to a novel stringy description of elementary particles characterized by a single compact (cyclic) world-line parameter playing the role of the particle internal clock. This clearly reveals an exact unified formulation of quantum and relativistic physics, potentially deterministic, fully falsifiable having no fine-tunable parameters, also proven in previous papers to be completely consistent with all known physics, from theoretical physics to condensed matter. New physics will be discovered by observing quantum phenomena with experimental time accuracy of the order of $10^{-21} \mathrm{sec}$.
\end{abstract}

Keywords Feynman Path Integral, Physics beyond Quantum Mechanics, Space-Time Geometry, Elementary Particles, Concept of Time

\section{Introduction}

The Feynman Path Integral (FPI) is one of the most important theoretical achievements of modern physics. It represents a mathematical formulation of Quantum Mechanics (QM) equivalent to the axiomatic one, as proven by Feynman [1], whose validity has been confirmed with impressing accuracy in countless experiments. We prove in App.(A) that the FPI, if correctly inquired in a bottom-up approach, unequivocally reveals an unedited nature of relativistic space-time beyond QM. It must be clear that the correctness of the ordinary mathematical formulations of quantum physics is absolutely not questioned in this paper, as well as the essence of relativity. We will pinpoint the physical principle at the origin of QM. The ordinary mathematical formulation of relativistic QM, such as the FPI formulation investigated here or QFT, is confirmed to be doubtlessly correct and indisputably exact in calculating observables, but it turns out to be an affective description emerging from

University of Camerino, Piazza Cavour 19F, 62032 Camerino, Italy. 
ultrafast cyclic relativistic space-time dynamics, at the base of wave-particle duality, which can not be directly detected with the present experimental temporal resolution.

\section{Cyclic Feynman Paths}

In the standard interpretation of the FPI the probability amplitude of a quantum particle to travel between two space-time points is given by the interference of all the paths - classical and non-classical - joining them. The probabilistic weight is given by the particle action $\mathcal{S}$ evaluated along the paths. Only a classical path is possible between two end-points, as long as the classical action is defined on the ordinary, implicitly non-compact, relativistic space-time. As pointed out by Feynman, the price to pay to reconcile QM with the Lagrangian formulation seems that to give a physical meaning to the paths not allowed by least action principle of classical mechanics. Nevertheless, Feynman himself, with his checkerboard model [2], tried to go beyond this interpretation by investigating the possibility to write the FPI as a discrete sum of paths, i.e. labeled by an integer number. If this is the case it would be possible to conceive a classical action whose minimization yields a numerable infinity of (degenerate) classical paths. For instance, in a compact (or cyclic) geometry, infinite, degenerate, classical paths are possible between two arbitrary endpoints, due to the Boundary Conditions (BCs): they would be labeled by an integer number (winding number).

In App.(A) - see demonstration - we rigorously prove that the FPI for a free scalar relativistic particle, here denoted by $\mathcal{Z}$, can be written as a discrete sum of cyclic paths according to the fundamental mathematical identity (natural units $\hbar=c=1$ )

$$
\mathcal{Z}=\int \mathcal{D}^{3} x e^{i \mathcal{S}} \equiv T_{C} \sum_{n^{\prime} \in \mathbb{Z}} \delta\left(\tau_{f}-\tau_{i}+n^{\prime} T_{C}\right)
$$

where $\mathcal{S}$ is the relativistic free particle action (defined in the ordinary noncompact space-time), $T_{C}=2 \pi / M$ is the particle Compton time defined in terms of the particle mass $M, \tau_{i}$ and $\tau_{f}$ are the initial and final word-line points of the particle evolution. The demonstration, given in App.(A) for a free multiparticle state, is based on widely accepted mathematical identities of second quantization and their direct consequences. By construction it can be generalized to interacting particles and fields, see par.(6), as well as to the functional formulation of the FPI [3-10], see also [11-19].

From a physical point of view, the FPI clearly reveals in eq.(1) something extremely important about the nature of space-time beyond QM. The integer index $n^{\prime}$ labeling the paths is manifestly a winding number. The Dirac deltas describe all the possible classical paths between arbitrary $\tau_{i}$ and $\tau_{f}$ on a compact world-line $\tau$ of compactification length $T_{C}$ and Periodic BCs (PBCs) that we name elementary world-cycle (or proper-cycle). 
In order to deal with this puzzling physical result the reader must consider that the parametrization of the particle evolution in terms of the world-cycle (rather than a non-compact world-line) is, after all, the real essence of the wave-particle duality and undulatory mechanics, see also par.(5). As stated by de Broglie in his seminal $\mathrm{PhD}$ thesis [20] at the origin of QM, "to each elementary particle with proper mass $M$, one may associate a periodic phenomenon of Compton periodicity", or, in Penrose words [21], "any stable massive particle behaves as a very precise quantum clock, which ticks away with Compton periodicity", and according to Einstein [22] "a clock is a periodic phenomenon so that what it happens in a period is identical to what happens in any other period". There is nothing wrong in describing quantum particles as intrinsic clocks ticking at Compton rates, this is implicitly done every time we use a wave function or a field in QM. Notice that massless particles such as photons or gravitons are "frozen clocks" [21] (infinite world-line compactification lenght $T_{C}=\infty$ ). In particular this element is useful to figure out how the ordinary causal structure of relativistic physics is preserved and how massive particle can propagate in space-time despite their compact world-cycles. Other elements will be give when we will introduce interactions in par.(6). In general we must always consider that: 1) it is a fact that the universe is solely constituted of elementary particles; 2 ) it is a fact that, according to the waveparticle duality, every elementary particle is a periodic phenomenon $[20,22$, $21]$; thus it must be true that physics can be consistently formulated in terms of elementary cycles.

Naively, the fact that each elementary free particle is a persistent periodic phenomenon does not mean that the world must be periodic in time as much as Newton's first law does not imply that everything moves in straight lines (persistent space-time periodicity means free particle). As for Newton's law our strategy is to clearly define the behavior of the isolated building-blocks of nature and then generalize to interactions between them, as we will see in par.(6) - a simple system of two periodic phenomena is already ergodic, when interactions are consider we will find the complexity of ordinary physics.

Even though the present paper is self-consistent, a wider view of the following description can be found in [3-19]. The reader should first convince himself about the absolute correctness of the mathematical demonstrations proposed here and in previous papers - the demonstrations are crosschecked and correct above any reasonable doubt as also certified by many peer-reviewed papers and rely on them for the non-trivial conceptual effort necessary to figure out such a radical new view of physics.

\section{Recovering the least action principle in QM}

The Feynman paths written as in eq.(1) are actually classical paths. They are the degenerate classical solutions, from $\tau_{i}$ to $\tau_{f}$, making stationary the action

$$
\mathcal{S}_{\text {Compact }} \doteq-\frac{1}{T_{C}} \oint_{T_{C}} d \tau,
$$


where the symbol $\oint_{T_{C}}$ means that the action is defined on the world-cycle - contrarily to $\mathcal{S}$ which is defined on the ordinary non-compact space-time. The classical variational principle can be eventually concealed with QM. The price to pay is to give up with the emphatically non-compact formulation of minkowskian space-time.

The resulting theory is exactly Elementary Cycles Theory (ECT) [3-19]. Here, for simplicity's sake, we have only considered PBCs but, in general, combinations of Neumann and Dirichlet BCs are allowed for a bosonic relativistic theory (without additional boundary terms), whereas for the fermionic action we have more complex BCs resulting in "twisted" periodic dynamics [6] (see also the zitterbewegung [23,24]). Different types of particles correspond to different compactified geometries. In fact, as long as the imposed BCs are allowed by the variational principle for that specific relativistic action, it is guaranteed that they do not break relativity, this is well-known in physics of the extra-dimensions, see e.g. $[25,26]$.

\section{Novel string description of elementary particles}

Among the various interpretations of new physics beyond QM allowed by eq.(1) here we will focus on a possible novel stringy description, based on ECT, that we name Elementary Cycles String Theory (ECST). In fact, $\mathcal{S}_{\text {Compact }}$ is manifestly a string action. It associates a closed string of novel type to each elementary particle. The idea of a stringy description of elementary particles is not new, of course, but surprisingly such a novel string theory emerging from the FPI is defined on a compact world-line (world-cycle) rather than on the two dimensional world-sheet of Ordinary String Theory (OST). According to eq.(1), see demonstration in App.(A), these novel relativistic strings, classical in the essence, constitute the fundamental quantum oscillators at the base of quantum fields.

From a historical point of view we know from Regge et al. that the good mathematical properties of OST originates from the compact parameter of the ordinary two-dimensional world-sheet. Actually, most of the mathematical beauty of OST is inherited by $\mathrm{ECT}^{1}$ as consequence of the compact world-line parameter [5]. Our result suggests that the non-compact world-line parameter of OST could be absolutely unnecessary to describe time evolution as soon as we take into account that elementary particles, the basic constituents of our universe, are the elementary clocks of nature according to QM. The whole physics can be formulated in terms of elementary cycles. On the other hand, a single compact world-line parameter as in ECST, contrarily to the ordinary two dimensional world-sheet, avoids problematic aspects of OST such as the proliferation of extra-dimensions. As we are going to see, in ECST

\footnotetext{
1 E.g., the vibrational harmonics coincides with the particle quantum excitations (Regge poles), the Fourier coefficients automatically satisfy Virasoro algebra and identify the Ladder operators of the ordinary Klein-Gordon field with implicit commutation relation (non second quantization is necessary) [5].
} 
the target space-time is in fact the ordinary four-dimensional minkowskian space-time, provided the contravariant compactification at Compton lengths encoding undulatory relativistic mechanics directly into the structure of the four-dimensional minkowskian geometry of relativity. Last but not least ECST is phenomenological predictive: we have seen the equivalence with the FPI and with all QM axioms [3-7]; the elementary cycles strings are the fundamental oscillator of ordinary QFT; ECT has been successfully applied to describe quantum phenomena in different fields, from theoretical physics to condensed matter [3-10].

\section{Elementary space-time cycles}

According to App.(A), see eq.(7), the FPI for the quantum evolution of a free particle in a generic inertial reference frame can be written as discrete sum of cyclic paths. In fact, by writing eq.(1) in covariant notation we get

$$
\mathcal{Z}=\int \mathcal{D}^{3} x e^{i \mathcal{S}} \equiv 2 \pi \sum_{n^{\prime} \in \mathbb{Z}} \delta\left(\omega_{\mu} \Delta x^{\mu}+2 \pi n^{\prime}\right) .
$$

where $\omega_{\mu}$ is the particle four-momentum (persistent, as we are in the free case); $\Delta x^{\mu}=x_{f}^{\mu}-x_{i}^{\mu}$ is the interval between the final and the initial spacetime points of the free particle evolution. In complete analogy with the rest frame description eq.(1), here the FPI describes space-time cycles of temporal recurrence $T=2 \pi / \omega$ and spatial recurrences $\lambda^{i}=2 \pi / k_{i}, i=1,2,3$ (these are global recurrences as we are in the free case, in the interaction case they are promoted to local quantities, as we will see below). These are, of course, the ordinary recurrences of QM. It is convenient to introduce the contravariant (global) four-vector $\lambda^{\mu}=\{T, \vec{\lambda}\}$ fixed by the (persistent) particle fourmomentum $\omega_{\mu}=\{\omega,-\vec{k}\}$ of the free particle through the Planck constant: $\omega_{\mu} \lambda^{\mu}=M T_{C}=2 \pi$. In fact, ${ }^{2}$ these space-time recurrences are obtained by Lorentz transforming the world-line Compton periodicity $T_{C}=2 \pi / M$.

The paths in the FPI eq.(3) are the classical degenerate solutions linking the final and initial space-time points $x_{i}{ }^{\mu}$ and $x_{f}{ }^{\mu}$ of the world-circle action $\left(\dot{x}^{\mu}(\tau)=d x^{\mu} / d \tau\right)$

$$
S_{\text {Compact }}=-\frac{1}{T_{C}} \oint_{\tau_{i}}^{\tau_{f}} d \tau \sqrt{\dot{x}^{\mu}(\tau) \dot{x}_{\mu}(\tau)} .
$$

For a free quantum particle of persistent momentum $\vec{k}$, the cyclic paths in the FPI eq.(3), i.e. the elementary cycles string vibrations, interfere constructively if the end points $x_{i}^{\mu}$ and $x_{f}^{\mu}$ of the particle evolution are along the ordinary classical-relativistic trajectory (the ordinary one in non-compact space-time),

\footnotetext{
2 According to the Lorentz transformations: we have $T_{C}=\gamma T-\gamma \vec{\beta} \cdot \vec{\lambda}$ where $\gamma=$ $1 / \sqrt{1-\vec{\beta}^{2}}$ is the Lorentz factor, so that $M T_{C}=\gamma M T-\gamma M \vec{\beta} \cdot \vec{\lambda}=\omega T-\vec{k} \cdot \vec{\lambda}=\omega_{\mu} \lambda^{\mu}=2 \pi$ , since $\omega_{\mu}=\{\gamma M,-\gamma M \vec{\beta}\}=\{\omega,-\vec{k}\}$.
} 
whereas the interference becomes more and more destructive as the sum over cyclic path is evaluated with one of the end points placed away from the classical trajectory. This corresponds a lower probability to observe the particle away from the classical trajectory, in full agreement - also numerical - with the ordinary probabilistic description of the FPI, [3-10]. Of course, eq.(3) in the in the non-relativistic classical limit reduces on a single Dirac delta over the particle classical path, as it can be easily proven by noticing that the spatial separation between the cyclic paths tends to infinity (in the semiclassical-limit of massive particles $|\vec{k}| \ll M$ so that $|\vec{k}| \rightarrow \infty$ and $T_{C} \rightarrow 0$ ).

Our result reveals that the Compton periodicity of elementary particles must be "super-imposed" to the minkowskian space-time geometry, exactly as in ECT. This is not a conjecture, it is an exact implication of the FPI, eq.(1), as well as of a long list of striking equivalencies too long to be mentioned here, [3-10]. Einstein intuition on his latest years - after Bell's work on hidden variables - was that the unification of relativity and QM would be possible by "super-imposing" (he used this term) some sort of boundary conditions to relativistic dynamics $[22,27]$. Relativity only defines the differential structure of space-time without concerning about "what happens at the boundary of space-time" whereas it was clear since its early days that QM was about BCs. From a mathematical point of view the introduction of a space-time boundary completes the Cauchy problem of relativity (differential structure plus BCs) solving simultaneously relativistic and quantum dynamics in a unified formulation of physics: the minkowskian metric of ECT preserves the ordinary differential structure of relativity whereas the controvariant BCs of ECT yield ordinary quantization (e.g. here we have seen the equivalence with the FPI) without breaking relativity (as long as the BCs are allowed by the variational principle for the relativistic action, as for the PBCs of the scalar relativistic particle investigated here).

\section{Interactions, QED and Gauge/Gravity correspondence}

In order to introduce interactions we must consider that the four-momentum of an interacting particle is no longer persistent as in the free case, but it varies locally according to the interaction scheme, see detailed proofs in [4]. In turns, the locally varying four-momentum of the interacting particle fixes locally its quantum space-time recurrences through the Planck constant. We must thus promote the global $\lambda^{\mu}$ of the free case to local space-time recurrences $\lambda^{\mu}(x)$ in order to describe the local four-momentum $\omega_{\mu}(x)$ of the interacting particle. In other words the elementary cycles strings associated to particles have locally modulated phases during interactions - this explain causality, as it can be established a "before" and a "after" the interaction: ECT does not imply that the world periodic!. Technically this can be done in terms of space-time geometrodynamics by locally deforming the elementary space-time cycles compactification lengths, provided that the invariant world-line recurrence is the particle Compton length; that is, by locally deforming the minkowskian space- 
time flat metric, similarly to general relativity. The interacting particle local classical action must be defined in a cyclic minkowskian space-time orbifold encoding the local modulations of space-time periodicity.

Two types of geometrodynamics are possible, see $[4,6,5]$ for a complete description and related formalism. The first type, the most obvious one, is characterized by local deformations of the metric tensor corresponding to a curved space-time. Of course this type of elementary cycles local deformations describes gravitational interaction exactly as in ordinary general relativity: e.g. it reproduces the ordinary clock rate modulations and ruler contractions encoded in a Schwarzschild metric.

Remarkably, the second type of deformations reveals a geometrodynamical origin of gauge interactions analogous to that of general relativity, in a unified view $[4,6,5]$, similar to original Weyl's proposal. Due to the compact nature of space-time in ECT it is possible to describe peculiar interaction schemes, i.e. particular local variations of space-time recurrences $\lambda^{\mu}(x)$ (local modulations of space-time phases), by locally transforming the metric tensor in such a way that the space-time boundary is locally rotated whereas the metric stays flat. The local "rotations" of the space-time boundary of ECT leaving the metric flat turns out the describe exactly gauge interactions. Notice in ECT such local rotations of the space-time boundary corresponds to particular local variations of space-time recurrences $\lambda^{\mu}(x)$ and thus to a peculiar type of interactions that turns out to be identical to gauge interactions. Notice that this type of transformations have no effect to ordinary quantum fields in which space-time has no boundary (they are in fact associated to Killing vector fields): this explains why in ordinary quantum fields it is not possible to observe the geometrodynamics associated to gauge interactions and, in turns, gauge invariance must be necessary postulated in ordinary QFT whereas in ECT gauge interactions can be deduced from space-time geometrodynamics.

Particularly simple is the abelian case (for the sake of simplicity here we only mention bosonic QED), [4]. This corresponds to local $U(1)$ rotations of compact space-time boundary of ECT. It results in local modulations of space-time phases (i.e. local modulations of space-time periodicities) formally identical to the ordinary minimal substitution $\omega_{\mu}(x)=\omega_{\mu}-e A_{\mu}(x)$, where $e$ is the charge of the particle and $A^{\mu}(x)$ is the gauge field - defined in terms of the peculiar Killing vector field associated to the local $U(1)$ rotation of the spacetime boundary. Generalizing the result of eq.(3), the FPI of bosonic QED turns out to describe a sum of cyclic paths with locally modulated phases. That is, the elementary cycles strings interacting under this peculiar interactions scheme have local modulations of phases $\omega_{\mu}(x)$ of ordinary electromagnetism. It is possible to prove the following identity for the FPI of the abelian gauge interaction (bosonic QED) [4]:

$$
\mathcal{Z}_{U(1)}=2 \pi \sum_{n^{\prime} \in \mathbb{Z}} \delta\left(\int_{x_{i}{ }^{\mu}}^{x_{f}^{\mu}}\left(\omega_{\mu}-e A_{\mu}(x)\right) d x^{\mu}+2 \pi n^{\prime}\right)
$$


The common geometrodynamical description of gauge and gravitational interactions allowed by ECT shed a new light on the problem of quantum gravity, $[4,6,5]$.

\section{Predictions of New Physics beyond QM}

It may be surprising that the scale of new physics deduced here from the FPI is at Compton scales (whereas it is common to assume that new fundamental physics is at Planck scale). But are we sure that we have observed everything about physics up to, say, the LHC energy scale? It is definitively true that we have explored physics up to the LHC energy scale, but we were only interested in probabilistic amplitudes, confirming the unquestionable correctness of QM probabilistic predictions of observables, according to Heisenberg, see also [33]. However, our result says something radically unedited, while keeping the mathematics of QM exactly valid at an effective level at every energy scale. The point is that in all these experiments we have not used ultra-accurate clocks. The new physics predicted here from the FPI can only be explored by means of ultra-accurate timekeepers able to detect and investigate the ultrafast cyclic dynamics of elementary particles.

Among a plethora of unexplored phenomena associated to ECT, we predict that new physics beyond QED will be detected by investigating quantum phenomena with time accuracy better than the electron Compton time: ${ }^{3}$ $T_{C}^{e l e c t}=\lambda_{C}^{\text {elect }} / c=8.09330093 \cdot 10^{-21} \mathrm{sec}$, and the electrons will reveal its fundamental cyclic string nature. It is reasonable to hope that this experimental time accuracy will be reached in the next future as the related technologies are already approaching the atto-second accuracy $[28,29]$.

If the time resolution of a quantum experiment is poorer than the Compton time scales of the particles involved, the ultra-fast cyclic dynamics can only be observed indirectly in a statistical way and the resulting probabilistic predictions are exactly described by the same mathematical rules of ordinary QM. QM emerges exactly as probabilistic, low time resolution description of the elementary cycles dynamics, as the outcomes of a rolling die can only be described probabilistically if the die is observed without ultra-fast timekeepers, see also [30-33,24]. We have rigorously proven in previous papers, [3-10], that the low time resolution description of ECT ultra-fast dynamics, at effective level is mathematically and phenomenologically equivalent to ordinary QM in all its fundamental aspects. For instance, besides the equivalence with the FPI eq.(3) proven in this paper, the exact equivalence between elementary cyclic dynamics and relativistic quantum dynamics has also been rigorously proven and crosschecked for all the fundamental identities of QM, including the canonical - Copenaghen axioms - formulation of QM and extended to QFT: all the postulates of QM as well the commutation relations has been mathematically

\footnotetext{
3 We have restricted this prediction to QED. E.g., the time resolution necessary to explore new physics beyond QM in electroweak dynamics is of the order $T_{C}^{E W} \sim 10^{-26}$ sec, corresponding to the periodicity associated to the electroweak energy scale.
} 
derived directly from the condition of intrinsic periodicity. ${ }^{4}$ ECT implies the same mathematical laws of ordinary QM $(A \Rightarrow B)$, the mathematical laws of QM correctly describes all quantum phenomena $(B \Rightarrow C)$, thus ECT correctly describes all quantum phenomena $(A \Rightarrow C)$.

Notice that in ECT there are not hidden variables of any sort: Bell's or similar no-go theorems cannot be invoked to rule out the equivalence with QM (the "hidden variable" would be the time parameter, which is not of course a hidden variable, whereas the PBCs is a strong element of non-locality conciliating the non-local aspects of QM and the request of locality of relativity) [30-33,24]. The Feynman paths, eq.(7), are on-shell paths: we have in fact a sum of Dirac deltas. This is clear indication of "onticity" as pointed out by 't Hooft. Actually, the evolution law of ECT can be alternatively written in terms of 't Hooft "ontic" coordinates as $\left|\tau_{i}\right\rangle \rightarrow \mid \tau_{f}$ MOD $\left.T_{C}\right\rangle$, or similarly for the free case as $\left|x_{i}{ }^{\mu}\right\rangle \rightarrow \mid x_{f}{ }^{\mu}$ MOD $\left.\lambda^{\mu}\right\rangle$, similarly to continuous periodic Cellular Automata ("particles on a circles") [30-33,24]. 't Hooft arguments suggests that the new physics beyond QM predicted by ECT is potentially deterministic, [3-10]. Also, the space-time of ECT can be imagined as formed of Compton elementary space-time cells, therefore we find a conceptual relationship with Quantum Time Crystals [34].

\section{Remarks}

The picture of new physics provided by ECT has exceptional beauty for many other aspects, besides the string description of elementary particles. It also supports many foundational ideas at the origin of the Kaluza-Klein theory, the Kaluza miracle, Holography, the AdS-CFT correspondence, Loop Quantum Gravity, just to mention a few; but it is extremely simple (harmonic), effective and refutable in addition to falsifiable. ${ }^{5}$ It doesn't involve extra parameters (rare case in modern theoretical physics typically characterized by fine-tuning plays) being based exclusively on the ordinary elements of relativity and the Planck constant to determine the space-time BCs.

We report some other essential facts about ECT. In more that ten years of serious researches, supported by solid mathematical demonstrations, and nearly 20 published papers on the topic, we can state without doubts that the cyclic (or more in general compact) formulation of space-time at Compton scales of ECT is completely consistent with all the theoretical foundations

\footnotetext{
${ }^{4}$ In short, integration by parts in compact dimensions implies boundary terms reproducing exactly the non-commutativities of ordinary QM, see full demonstrations in [3-10].

5 An apparently unbearable hypothesis such as compact relativistic time at Compton scales can be proven to be either absolutely correct or completely inconsistent with ordinary physics, but notice that it has passed quite a few of peer-reviews [3-19] where the many demonstrations of its full consistence are scientifically certified. All this is sign, at the very least, that the matter is not so obvious. As Feynman used to say: "Doubt is clearly a value in science. It is important to doubt and the doubt is not a fearful thing, but a thing of great value". This paper want to promote in the scientific community a serious discussion about the hypothesis of compact space-time, as really deserved and on scientific arguments.
} 
and phenomenology of both quantum physics and relativity (special and general), [3-10]. We have an unedited deep relationship between Feynman's and de Broglie's based interpretation of QM; between the "new" and the "old" formulations of QM. Another remarkable result proven mainly in [5] is that in ECT the "quantum to classical correspondence" at the base of the AdS/CFT correspondence turns out to be an exact mathematical identity rather than a conjecture: consider for instance the equivalence between quantum particles and classical strings presented in this paper. ECT is dual to an extra-dimensional theory where the compact world-line plays the role of a "virtual extra-dimension" with interesting insights into the Kaluza's miracle, whereas the common geometrodynamical description of gravitational and gauge interactions given above, see the extended proofs in [4], is actually a Gauge/Gravity correspondence [5]. Since, as we have seen, interactions, and the consequent local modulations of space-time recurrences, can be equivalently described as deformations of the compact space-time boundary, the theory is manifestly holographic $[4,6,5]$ (the bulk physics is determined by the shape of the boundary). The success of ECT is not limited to theoretical physics but, for instance, has been confirmed also in condensed matter where it has produced exceptional results. ECT has been successfully applied to infer all the fundamental aspects of superconductivity and graphene physics, in a striking mathematical way and for the first time directly from a first physical principle (intrinsic periodicity) rather than from empirical models (e.g., the BCS model), [8,9].

\section{Conclusions}

The equivalence, proven in this paper in a bottom-up approach, between the FPI evolution and elementary space-time cyclic dynamics is such a remarkable and striking result that it can not be classified as a mere mathematical coincidence. On the contrary it imposes us a serious reconsideration about the fundamental nature of relativistic space-time, especially if we take into account all the others crosschecked and peer-reviewed mathematical and phenomenological equivalencies with ordinary physics already proven in previous papers [3-10], see also [11-19], which in turns represents only the tip of the iceberg of possible new physics beyond QM. All this confirms that the intrinsically compact (cyclic) nature of space-time is de facto a conceptually legitimated, mathematically correct, phenomenologically predictive hypothesis for a potentially deterministic [30-33,24], unified formulation of quantum and relativistic physics $[3,6,7,23]$. Despite its radical unconventionality ECT has given more than sufficient scientific evidences to allow us to claim a new viable scenario in fundamental physics. It clearly predicts, without fine-tunings or hiddenvariables of any sort, that new physics will be observed by investigating quantum phenomena with time precision better that $10^{-21} \mathrm{~s}$. With sufficiently accurate ultra-fast timekeepers elementary particles will reveal their fundamental string nature, characterized by a one-dimensional world-cycle rather than a two-dimensional world-sheet, manifestation of the intrinsically cyclic nature 
of relativistic space-time. "God play dice?" Our result solves the quantum dilemma in this way: "God has no fun playing dice", having infinite accuracy in time He would always be able to predict the outcomes.

\section{A Counting the Feynman Paths (Proof)}

We prove eq.(1): the ordinary FPI of QM can be explicitly written as a discrete sum of paths, labelled by an integer number. Let us consider the FPI for a free relativistic scalar particle of momentum $\vec{k}$ traveling between $x_{i}{ }^{\mu}$ and $x_{f}{ }^{\mu}$. Assuming that $t_{i}$ to $t_{f}$ are the initial and final time of the evolution, respectively, the ordinary FPI is defined as $\mathcal{Z}=\int \mathcal{D}^{3} x e^{i \mathcal{S}\left[t_{f} ; t_{i}\right]}$, where $\mathcal{S}^{F r e e}\left[t_{f} ; t_{i}\right]=\int_{t_{i}}^{t_{f}} \mathcal{L} d t$ is the relativistic action and $\mathcal{L}=\overrightarrow{\mathcal{P}} \cdot \vec{x}-\mathcal{H}$ is the free particle classical Lagrangian defined by the Hamiltonian and momentum operators, $\mathcal{H}$ and $\overrightarrow{\mathcal{P}}$, respectively.

We perform the ordinary time slicing. In the limit $N \rightarrow \infty$ we have

$$
\mathcal{Z}=\lim _{N \rightarrow \infty} \int\left(\prod_{j=0}^{N-1} d^{3} x_{j}\right) \mathcal{U}_{f, N-1} \cdots \mathcal{U}_{1, i}
$$

where the elementary Feynman space-time evolutions are

$$
\mathcal{U}_{m+1, m}=\left\langle\Phi_{\vec{k}}\left|e^{-i\left[\mathcal{H}(\vec{k}) \Delta t_{m}-\overrightarrow{\mathcal{P}} \cdot \Delta \vec{x}_{m}\right]}\right| \Phi_{\vec{k}}\right\rangle
$$

the time slice $\Delta t_{m}=t_{m+1}-t_{m}$ is associated to spatial intervals $\Delta \vec{x}_{m}=\vec{x}_{m+1}-\vec{x}_{m}$ whereas $\left|\Phi_{\vec{k}}\right\rangle$ is the multiparticle state ${ }^{6}$ of momentum $\vec{k}$ associated to the particle, defined in terms of the local energy eigenstates $\left|n_{\vec{k}}\right\rangle$. In other words, since we are considering a relativistic scalar particle, $\left|\Phi_{\vec{k}}\right\rangle$ can be regarded as the single component of momentum $\vec{k}$ of the second quantized Klein-Gordon field state $|\Phi\rangle$. It must have both positive and negatives frequencies $k_{0}= \pm \omega(\vec{k}) / c$ as a consequence of the quadratic relativistic dispersion relation $k_{0}^{2}=\vec{k}^{2}+M^{2}$. It is well known that the second quantization of each field component yields the normal ordered energy spectrum $\omega_{\tilde{n}_{\vec{k}}}(\vec{k}):=: \tilde{n}_{\vec{k}} \omega(\vec{k})$ with $\tilde{n}_{\vec{k}} \in \mathbb{N}$ for both the positive and negative frequencies. As usual in the FPI analysis we will always assume normal ordering (:=:). So, when both positive and negative frequencies of $|\Phi\rangle$ are considered, the energy spectrum of the Hamiltonian operator $\mathcal{H}(\vec{k})$ in eq. $(5)$ can be equivalently written as $\mathcal{H}(\vec{k})\left|n_{\vec{k}}\right\rangle:=: n_{\vec{k}} \omega(\vec{k})\left|n_{\vec{k}}\right\rangle$, with $n_{\vec{k}} \in \mathbb{Z}$. Through the quadratic relativistic dispersion relation the energy spectrum fixes the momentum spectrum of $\left|\Phi_{\vec{k}}\right\rangle$ as well. It follows that $\overrightarrow{\mathcal{P}}\left|n_{\vec{k}}\right\rangle:=: n_{\vec{k}} \vec{k}\left|n_{\vec{k}}\right\rangle$, with $n_{\vec{k}} \in \mathbb{Z} .^{7}$

So far we have essentially recalled widely accepted identities of second quantization and theirs direct consequences. We evaluate eq.(5) by using the energy and momentum spectra associated to the Hamiltonian and momentum operators of $\left|\Phi_{\vec{k}}\right\rangle$ described above, then we apply the Poisson summation $\sum_{n \in \mathbb{Z}} e^{-i n y}=2 \pi \sum_{n^{\prime} \in \mathbb{Z}} \delta\left(y+2 \pi n^{\prime}\right)$, where $n, n^{\prime} \in \mathbb{Z}$. We find that a sum of Dirac deltas is associated to the elementary Feynman evolutions eq.(5),

$$
\begin{aligned}
\mathcal{U}_{m+1, m} & =\sum_{n_{m} \in \mathbb{Z}} e^{-i n_{m}\left[\omega(\vec{k}) \Delta t_{m}-\vec{k} \cdot \Delta \vec{x}_{m}\right]} \\
& =2 \pi \sum_{n_{m}^{\prime} \in \mathbb{Z}} \delta\left(\omega(\vec{k}) \Delta t_{m}-\vec{k} \cdot \Delta \vec{x}_{m}+2 \pi n_{m}^{\prime}\right) .
\end{aligned}
$$

${ }^{6}$ For simplicity's sake, we address it as quantum particle of momentum $\vec{k}$. It is the result of the field component $\Phi_{\vec{k}}$ second quantization. It must not be confused with the single particle of ordinary QM.

7 Notice that the negative values of $n_{\vec{k}}$ provide a correct description of the negative negative energy eigenmodes and their momenta. For instance, in ordinary QFT the annihilation operator is defined in terms of the creation operator as $\mathbf{a}^{\dagger}(\vec{k})=\mathbf{a}(-\vec{k})$. 
We plug this result in the FPI, eq.(4), and we apply the Dirac delta property $\int d^{3} x_{m} \delta\left(\vec{x}_{m+1}-\right.$ $\left.\vec{x}_{m}\right) \delta\left(\vec{x}_{m}-\vec{x}_{m-1}\right)=\delta\left(\vec{x}_{m+1}-\vec{x}_{m-1}\right)$. We finally find that the ordinary FPI of a free relativistic quantum particle of momentum $\vec{k}$ can be explicitly expressed as the sum of paths, represented by Dirac deltas, and labeled by the integer number $n^{\prime}$ :

$$
\mathcal{Z}=2 \pi \sum_{n^{\prime} \in \mathbb{Z}} \delta\left(\omega(\vec{k})\left(t_{f}-t_{i}\right)-\vec{k} \cdot\left(\vec{x}_{f}-\vec{x}_{i}\right)+2 \pi n^{\prime}\right) .
$$

Eq.(3) is obtained by writing this result in covariant notation whereas our thesis eq.(1) refers to an observer in the particle reference frame.

\section{References}

1. R. P. Feynman, The principle of least action in quantum mechanics. Laurie M. Brown, 1939.

2. R. P. Feynman and A. R. Hibbs, Quantum mechanics and path integrals. International series in pure and applied physics. McGraw-Hill, New York, NY, 1965.

3. D. Dolce, "Compact Time and Determinism for Bosons: foundations," Found. Phys. 41 (2011) 178-203, 0903.3680v5.

4. D. Dolce, "Gauge Interaction as Periodicity Modulation," Annals Phys. 327 (2012) 1562-1592, 1110.0315.

5. D. Dolce, "Classical geometry to quantum behavior correspondence in a Virtual Extra Dimension," Annals Phys. 327 (2012) 2354-2387, 1110.0316.

6. D. Dolce, "Elementary spacetime cycles," Europhys. Lett. , 102 (2013) 31002, 1305.2802.

7. D. Dolce, "Unification of Relativistic and Quantum Mechanics from Elementary Cycles Theory," Electron. J. Theor. Phys. 12 (2016) 29-86, 1606.01918.

8. D. Dolce and A. Perali, "The role of quantum recurrence in superconductivity, carbon nanotubes and related gauge symmetry breaking," Found.Phys. 44 (Sept., 2014) 905-922, 1307.5062.

9. D. Dolce and A. Perali, "On the Compton clock and the undulatory nature of particle mass in graphene systems," EPJ Plus 41 (Jan., 2015) 130, 1403.7037.

10. D. Dolce, "Clockwork Quantum Universe," 1111.3319. IV Prize, QFXi contest (2011).

11. D. Dolce, Introduction to the Quantum Theory of Elementary Cycles, pp. 93-135. IMPERIAL COLLEGE PRESS, Apr, 2016. 1707.00677.

12. D. Dolce, "AdS/CFT as classical to quantum correspondence in a Virtual Extra Dimension," PoS ICHEP2012 (2013) 478, 1309.2646.

13. D. Dolce and A. Y. Khrennikov, "Quantum Mechanics from Periodic Dynamics: the bosonic case," AIP Conf. Proc. 1232 (2010) 222-227, 1001.2718.

14. D. Dolce, "de Broglie Deterministic Dice and Emerging Relativistic Quantum Mechanics," J. Phys.: Conf. Ser. 306 (2011) 10. 1111.3319.

15. D. Dolce, J. G. Hartnett, and P. C. Abbott, "Deterministic Quantization by Dynamical Boundary Conditions," AIP Conf. Proc. 1246 (2010) 178-181, 1006.5648.

16. D. Dolce and A. Perali, "Testing cellular automata interpretation of quantum mechanics in carbon nanotubes and superconductivity," Journal of Physics: Conference Series 626 (Jul, 2015) 012062. 1602.09023.

17. D. Dolce, "Intrinsic periodicity: the forgotten lesson of quantum mechanics," J. Phys.: Conf. Ser. 442 (2013) 012048, 1304.4167.

18. D. Dolce, "Elementary cycles of time," EPJ Web Conf. 58 (2013) 01018, 1306.0579.

19. D. Dolce, "On the intrinsically cyclic nature of space-time in elementary particles," $J$. Phys.: Conf. Ser. 343 (2012) 012031, 1206.1140.

20. L. d. Broglie Phil. Mag. 47 (1924) 446.

21. R. Penrose, Cycles of Time. An Extraordinary View of The Universe, ch. 2.3. Knopf, New York, 2011. 
22. A. Einstein, "Bietet die Feldtheorie MöglishKeiten für Lösung des Quantenproblems?," S.B. Press. Aked. Wiss. 33 (1923).

23. D. Hestenes, "The zitterbewegung interpretation of quantum mechanics," Foundations of Physics 20 (1990) 1213-1232.

24. C. Wetterich, "Probabilistic cellular automata for interacting fermionic quantum field theories," Nuclear Physics B 963 (Feb, 2021) 115296. 2007.06366.

25. C. Csaki, J. Hubisz, and P. Meade, "TASI lectures on electroweak symmetry breaking from extra dimensions," in Theoretical Advanced Study Institute in Elementary Particle Physics: Physics in $D \geqq 4$, pp. 703-776. 10, 2005. hep-ph/0510275.

26. M. Henneaux and C. Teitelboim, Quantization of gauge systems. 1992.

27. A. Pais, Subtle is the Lord: The science and the life of Albert Einstein. Oxford University Press, 1982

28. S. L. Campbell, R. B. Hutson, G. E. Marti, A. Goban, N. Darkwah Oppong, R. L. McNally, L. Sonderhouse, J. M. Robinson, W. Zhang, B. J. Bloom, and et al., "A fermi-degenerate three-dimensional optical lattice clock," Science 358 (Oct, 2017) $90 Đ 94$.

29. A. Föhlisch, P. Feuler, F. Hennies, A. Fink, D. Manzel, D. Sanchez-Portal, P.-M. Echenique, and W. Wurth, "Direct observation of electron dynamics in the attosecond domain," Nature 436 (July, 2005) 373-376.

30. G. 't Hooft, "How Does God Play Dice? (Pre-)Determinism at the Planck Scale," hep-th/0104219.

31. G. t. Hooft, "The Cellular Automaton Interpretation of Quantum Mechanics. A View on the Quantum Nature of our Universe, Compulsory or Impossible?," 1405.1548.

32. G. t Hooft, "Explicit construction of local hidden variables for any quantum theory up to any desired accuracy," 2021. 2103.04335.

33. G. t Hooft, "Fast vacuum fluctuations and the emergence of quantum mechanics," 2020. 2010.02019.

34. F. Wilczek, "Quantum Time Crystals," Phys.Rev.Lett. 109 (2012) 160401, 1202.2539. 